\title{
Note on the equi-ultimate boundedness of solutions of $\boldsymbol{x}^{\prime}=F(t, x)$
}

\author{
By \\ Taro Yoshizawa \\ (Received October 11, 1958)
}

In the papers [1], [2] and [3]* we have discussed a necessary and sufficient condition for the uniform boundedness and a necessary and sufficient condition for the uniform ultimate boundedness. In this paper we will discuss the equi-ultimate boundedness. Now we consider a system of differential equations,

$$
\frac{d x}{d t}=F(t, x)
$$

where $x$ denotes an $n$-dimensional vector and $F(t, x)$ is a given vector field which is defined and continuous in the domain

$$
\Delta: \quad 0 \leqq t<\infty, \quad\|x\|<\infty .
$$

The norm $\|a\|$ of a vector $a=\left(a_{1}, a_{2}, \cdots, a_{n}\right)$ represents $\sqrt{\sum_{i=1}^{n} a_{i}^{2}}$. Unless otherwise stated, we adopt the notations and the definitions in [1].

The following lemma is clearly true.

Lemma 1. If the solutions of (1) are equi-ultimately bounded, they are equi-bounded.

At first we will prove the two following lemmas.

Lemma 2. We assume that every solution of (1) is unique for the Cauchy-problem. Then if the solutions of (1) are equi-ultimately bounded, they are locally uniformly ultimately bounded.

Proof. We say that the solutions of (1) are locally uniformly ultimately bounded when for points $(\bar{t}, \bar{x})$ of a suitable neighborhood

\footnotetext{
* Numbers in [] refer to the bibliography at the end of the paper.
} 
of the point $\left(t_{0}, x_{0}\right)$ we can choose a positive constant as $T(\bar{t}, \bar{x})$ such that for a certain positive number $B$ independent of the particular solution we have $\|x(t ; \bar{x}, \bar{t})\|<B$ for $t>\bar{t}+T(\bar{t}, \bar{x})$. Now we assume that $\left\|x_{0}\right\| \leqq \alpha$ and we discuss in the region such as $\|x\| \leqq \beta$, where $\beta(>\alpha)$ is a constant. Since the solutions are equi-ultimately bounded, $T$ connected with $x\left(t ; x_{0}, t_{0}\right)$ is determined depending only on $t_{0}$ and $\beta$. We represent it by $T\left(t_{0}\right)$. Now we suppose that $T(t)$ is not bounded in any neighborhood of $\left(t_{0}, x_{0}\right)$. Then there exists a sequence of points $\left(t_{m}, x_{m}\right)$ such that

$$
\left(t_{m}, x_{m}\right) \rightarrow\left(t_{0}, x_{0}\right) \quad(m \rightarrow \infty)
$$

and $T\left(t_{m}\right)$ connected with $x\left(t ; x_{m}, t_{m}\right)$ tends to infinity as $m \rightarrow \infty$. By the continuity of the initial value, for a sufficiently large $m$, the solution $x\left(t ; x_{m}, t_{m}\right)$ intersects the hyperplane $t=t_{0}$ and besides $x\left(t_{0} ; x_{m}, t_{m}\right)$ is near $x_{0}$. Therefore it is sufficient that we consider the solutions issuing from the set $\left[t=t_{0},\|x\| \leqq \beta\right]$ to the right. $T$ connected with these solutions is $T\left(t_{0}\right)$. This contradicts that $T\left(t_{m}\right) \rightarrow \infty$. The proof is complete.

Lemma 3. We assume that every solution of (1) is unique for the Cauchy-problem. Then if the solutions of (1) are uniformly bounded and ultimately bounded, they are equi-ultimately bounded.

Proof. Let $\bar{B}$ be the bound for $B$ by the uniform boundedness, that is to say, for every solution $x\left(t ; x_{0}, t_{0}\right)$ such as $\left\|x_{0}\right\| \leqq B$ we have $\left\|x\left(t ; x_{0}, t_{0}\right)\right\| \leqq \bar{B}(\bar{B}>0)$. By the ultimate boundedness, we have $\left\|x\left(t ; x_{0}, t_{0}\right)\right\|<B$ for $t>t_{0}+T\left(t_{0}, x_{0}\right)$. We denote this $T\left(t_{0}, x_{0}\right)$ by $T_{0}$. We can choose suitable $t_{1}$ and $B^{\prime}$, where $t_{1}>t_{0}+T_{0}$ and we have $\left\|x\left(t_{1} ; x_{0}, t_{0}\right)\right\|=B^{\prime}<B$. We put $t_{1}=t_{0}+\tau_{0}$. On the hyperplane $t=t_{1}$ we take a neighborhood $V$ of the point $x\left(t_{1} ; x_{0}, t_{0}\right)$ such as

$$
V\left(x\left(t_{1} ; x_{0}, t_{0}\right)\right) \subset E\{x:\|x\| \leqq B\} .
$$

By the uniqueness of the solution, for this $V$ there exists a neighborhood $U\left(x_{0}\right)$ of the point $x_{0}$ on $t=t_{0}$ such that we have $x\left(t_{1} ; x, t_{0}\right) \in V$ for $x \in U$. Then for this solution we have $\left\|x\left(t ; x, t_{0}\right)\right\|$ $\left\langle\bar{B}\right.$ for $t>t_{1}=t_{0}+\tau_{0}$. Now for each point $x$ such as $\|x\| \leqq$ $\alpha\left(\alpha>0\right.$ : arbitrary) on $t=t_{0}$ we take such a neighborhood $U_{x}$ as the above-mentioned. Since the set $E_{\alpha}\{x:\|x\| \leqq \alpha\}$ is compact, it is covered by finite numbers of $U_{x}$, say $U_{i}(1 \leqq i \leqq k)$. If we put 
Note on the equi-ultimate boundedness of solutions of $x^{\prime}=F(t, x) \quad 213$

$$
T=\max _{i} \tau_{i},
$$

where $\tau_{i}(1 \leqq i \leqq k)$ are determined by $U_{i}, T$ depends only on $t_{0}$ and $\alpha$. And we have

$$
\left\|x\left(t ; x, t_{0}\right)\right\|<\bar{B} \quad\left(x \in E_{\alpha}\right)
$$

for $t>t_{0}+T$. Therefore the solutions of (1) are equi-ultimately bounded for the bound $\vec{B}$.

Theorem 1. We assume that $F(t, x)$ in (1) belongs to the class $C_{0}$ with respect to $x$. In order that the solutions of (1) are uniformly bounded and ultimately bounded (consequently they are equi-ultimately bounded by Lemma 3), it is necessary and sufficient that there exist two positive continuous functions $\varphi(t, x)$ and $\psi(t, x)$ satisfying the following conditions in the domain

$\tilde{\Delta}: \quad 0 \leqq t<\infty,\|x\| \geqq B_{0} \quad\left(B_{0}\right.$ be a certain positive constant $):$

$1^{\circ} \quad \alpha(\|x\|) \leqq \mathcal{P}(t, x) \leqq \beta(\|x\|)$, where $\alpha$ and $\beta$ are positive continuous functions and $\alpha(\|x\|) \rightarrow \infty$ as $\|x\| \rightarrow \infty$,

$2^{\circ} \quad \gamma(\|x\|) \leqq \psi(t, x)$, where $\gamma$ is a positive continuous function,

$3^{\circ}$ both $\phi(t, x)$ and $\psi(t, x)$ belong to the class $C_{0}$ with respect to $(t, x)$

$$
\begin{array}{ll}
4^{\circ} & D_{F} \mathcal{P}(t, x)=\varlimsup_{h \rightarrow 0} \frac{1}{h}\{\mathcal{P}(t+h, x+h F)-\mathcal{P}(t, x)\} \leqq 0 \quad \text { (cf. [1]), } \\
5^{\circ} & D_{F} \psi(t, x) \leqq-\psi(t, x) .
\end{array}
$$

Proof. At first we will show that the condition is sufficient. The uniform boundedness of solutions is verified by the existence of $\varphi(t, x)$ (cf. [1]). For the solution $x\left(t ; x_{0}, t_{0}\right)$ such as $B_{0}<\left\|x_{0}\right\|$ $\leqq K$, there exists $\delta>0$ such that $\left\|x\left(t ; x_{0}, t_{0}\right)\right\| \leqq \delta(K)$, where this $\delta$ is determined by the uniform boundedness of the solutions. Now we assume that $B_{0}<\left\|x\left(t ; x_{0}, t_{0}\right)\right\| \leqq \delta(K)$ for all $t$. Consider the function $\psi\left(t, x\left(t ; x_{0}, t_{0}\right)\right)$ and then by the condition $2^{\circ}$

$$
\psi\left(t, x\left(t ; x_{0}, t_{0}\right)\right) \geqq \lambda>0 \quad(\lambda \text { : a suitable constant }) .
$$

On the other hand, by the condition $5^{\circ}$, we have

$$
\psi\left(t, x\left(t ; x_{0}, t_{0}\right)\right)-\psi\left(t_{0}, x\left(t_{0} ; x_{0}, t_{0}\right)\right) \leqq-\lambda\left(t-t_{0}\right) .
$$

Therefore we have $\psi\left(t, x\left(t ; x_{0}, t_{0}\right)\right)<0$ for a sufficiently large $t$. This contradicts (2). Hence for some $t$, say $t_{1}$, we have 
$\left\|x\left(t_{1} ; x_{0}, t_{0}\right)\right\|=B_{0}$. Then by the uniform boundedness, we have

$$
\left\|x\left(t ; x_{0}, t_{0}\right)\right\|<\delta\left(B_{0}\right)
$$

when $t>t_{1}$. Therefore for a suitable $T_{0}$ depending only on $t_{0}$ and $K$, if $t>t_{0}+T_{0}$, we have

$$
\left\|x\left(t ; x_{0}, t_{0}\right)\right\|<\delta\left(B_{0}\right) .
$$

Namely the solutions of (1) are equi-ultimately bounded for the bound $\delta\left(B_{0}\right)$.

Next we will prove that the condition is necessary. As we have proved in the paper [3], the existence of the function $\mathcal{P}(t, x)$ is derived from the uniform boundedness of solutions. Now we suppose that the solutions of (1) are equi-ultimately bounded for the bound $B$. We consider a continuous function $G(\eta)$ for $\eta \geqq 0$ which is defined as follows; namely

$$
G(\eta)= \begin{cases}\eta-B & (\eta \geqq B) \\ 0 & (0 \leqq \eta<B) .\end{cases}
$$

Since the solutions are locally uniformly ultimately bounded by Lemma 2, $T(t, x)$ connected with the ultimate boundedness can be considered as a continuous increasing function of $(t,\|x\|)$. Now we put

$$
\psi(t, x)=\sup _{\tau}\left[G(\|x(t+\tau ; x, t)\|) e^{\tau} ; 0 \leqq \tau\right] .
$$

Then it is clear that we have

$$
G(\|x\|) \leqq \psi(t, x) .
$$

And $G(\|x\|)$ is a positive function for $\|x\| \geqq B_{0}$, where $B_{0}>B$, and hence $\psi(t, x)$ has the property $2^{\circ}$. Particularly from the uniform boundedness and the property of $G(\eta)$, we can easily see that the inequality

$$
\psi(t, x) \leqq G(\delta\|x\|)) e^{T(t,\|x\|)}
$$

holds good.

Since we have

$$
\left|G(\eta)-G\left(\eta^{\prime}\right)\right| \leqq\left|\eta-\eta^{\prime}\right|,
$$

the condition $3^{\circ}$ can be proved in the same way as one in the proof of Theorem 2 in [3]. 
Note on the equi-ultimate boundedness of solutions of $x^{\prime}=F(t, x) \quad 215$

Let $\left(t^{\prime}, x^{\prime}\right)$ be a point on the solution issuing from $(t, x)$ to the right. Then we have

$$
\begin{aligned}
\psi\left(t^{\prime}, x^{\prime}\right) & =G\left(\left\|x\left(t^{\prime}+\tau^{\prime} ; x^{\prime}, t^{\prime}\right)\right\|\right) e^{\tau^{\prime}} \\
& =G(\|x(t+\tau ; x, t)\|) e^{\tau^{\prime}} \quad\left(t^{\prime}+\tau^{\prime}=t+\tau\right) \\
& =G(\|x(t+\tau ; x, t)\|) e^{\tau} \cdot e^{\tau^{\prime-\tau}} \\
& \leqq \psi(t, x) e^{\tau^{\prime}-\tau} .
\end{aligned}
$$

Since if $t^{\prime}=t+h, \tau^{\prime}-\tau$ is equal to $-h$, we have

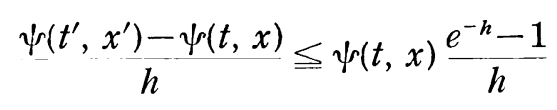

from which we can obtain $D_{F} \psi \leqq-\psi$.

From the proof of Theorem 1, it is clear that the function $\psi(t, x)$ satisfies the inequality

$$
G(\|x\|) \leqq \psi(t, x) \leqq G(\delta(\|x\|)) e^{T(t .\|x\|)} .
$$

Moreover $G(\|x\|) \rightarrow \infty$ as $\|x\| \rightarrow \infty$. Since there exists $\mu(\|x\|)$ such as $T(t,\|x\|) \leqq \mu(\|x\|)$ when the solutions of (1) are uniformly ultimately bounded, there exist $\tilde{\alpha}(\|x\|)$ and $\tilde{\beta}(\|x\|)$ for which we have

$$
\tilde{\alpha}(\|x\|) \leqq \psi(t, x) \leqq \tilde{\beta}(\|x\|)
$$

and $\tilde{\alpha}(\|x\|) \rightarrow \infty$ as $\|x\| \rightarrow \infty$. From (6) and the condition $5^{\circ}$ it follows that the solutions of (1) are uniformly bounded. Therefore it is right that the condition in order to be uniformly bounded and uniformly ultimately bounded is only the existence of $\psi(t, x)$.

The condition for the mere equi-ultimate boundedness is as follows.

Theorem 2. We assume that $F(t, x)$ in (1) belongs to the class $C_{0}$ with respect to $x$. Then in order that the solutions of (1) are equi-ultimately bounded, it is necessary and sufficient that there exist a positive number $B$ and $a$ non-negative continuous function $\varphi(t, x)$ satisfying the following conditions in the domain $\Delta:$ namely

$1^{\circ} \quad \alpha(\|x\|) \leqq \varphi(t, x)$ for $\|x\| \geqq B$, where $\alpha(\eta)$ is a continuous function which is positive increasing for $\eta>B$ and tends to infinity as $\eta \rightarrow \infty$, 
$2^{\circ} \quad \mathcal{P}(t, x)$ belongs to the class $C_{0}$ with respect to $(t, x)$, $3^{\circ} \quad D_{F} \varphi(t, x) \leqq-\varphi(t, x)$.

Proof. We will show that the condition is sufficient. Namely we will prove that the solutions are equi-ultimately bounded for the bound $\bar{B}$ such as $\bar{B}>B$. It is clear that the solutions are bounded. Now if we put

$$
\psi(t, x)=e^{t} \mathcal{P}(t, x) .
$$

$\psi^{\prime}(t, x)$ satisfies the following conditions:

$1^{\circ \prime} \quad \alpha(\|x\|) e^{t} \leqq \psi(t, x) \quad$ for $\|x\| \geqq B$,

$2^{\circ \prime} \psi^{\prime}(t, x)$ belongs to the class $C_{0}$ with respect to $(t, x)$,

$3^{\circ \prime} \quad D_{F} \psi(t, x) \leqq 0$.

We suppose that for a certain solution, say $x\left(t ; x_{0}, t_{0}\right)\left(\left\|x_{0}\right\| \leqq K\right)$, we have

$$
\left\|x\left(t_{m} ; x_{0}, t_{0}\right)\right\|>\bar{B}
$$

for the sequence $\left\{t_{m}\right\}$ such as $t_{m} \rightarrow \infty(m \rightarrow \infty)$. Then we have

$$
\psi\left(t_{m}, x\left(t_{m} ; x_{0}, t_{0}\right)\right) \geqq \alpha(\bar{B}) e^{t_{m}} .
$$

On the other hand, by the conditions $2^{\circ \prime}$ and $3^{\circ \prime}$, we have

$$
\psi\left(t_{m}, x\left(t_{m} ; x_{0}, t_{0}\right)\right) \leqq \psi\left(t_{0}, x\left(t_{0} ; x_{0}, t_{0}\right)\right) .
$$

If we put

$$
\max _{\|x\| \leqq K} \psi\left(t_{0}, x\right)=\beta\left(t_{0}\right)
$$

we have

$$
\alpha(\bar{B}) e^{t_{m}} \leqq \beta\left(t_{0}\right)
$$

Since $\alpha(\bar{B})>0$ and $t_{m} \rightarrow \infty \quad(m \rightarrow \infty)$, there arises a contradiction. Therefore we can see that the solutions are equi-ultimately bounded for the bound $\bar{B}$. The $T$ connected with the equi-ultimate boundedness is determined by (7).

Inversely we suppose that the solutions are equi-ultimately bounded for the bound $B$. In the same way as one in Theorem 1 , considering the function $G(\eta)$, we put

$$
\varphi(t, x)=\sup _{\tau}\left[G(\|x(t+\tau ; x, t)\|) e^{\tau} ; 0 \leqq \tau\right] .
$$

By Lemma 1, the solutions are equi-bounded and consequently 
Note on the equi-ultimate boundedness of solutions of $x^{\prime}=F(t, x) \quad 217$

they are locally uniformly bounded and moreover by Lemma 2, they are locally uniformly ultimately bounded. Therefore in the same way as one in Theorem 1 , we can see that this $\varphi(t, x)$ is the desired.

\section{BIBLIOGRAPHY}

[1] T. Yoshizawa, "Note on the boundedness and the ultimate boundedness of solutions of $x^{\prime}=F(t, x)$ ", These Memoirs, Vol. 29 (1955) pp. 275-291.

[2] T. Yoshizawa, "Appendix to the paper "Note on the boundedness and the ultimate boundedness"”, These Memoirs, Vol. 30 (1957) pp. 91-103.

[3] T. Yoshizawa, "On the necessary and sufficient condition for the uniform boundedness of solutions of $x^{\prime}=F(t, x)$ ", ibid., pp. 217-226. 